\title{
Evolution of Contact Resistance and Coupling Loss in Prototype ITER PF NbTi Conductors Under Transverse Cyclic Load
}

\author{
A. Nijhuis, Yu. Ilyin, W. Abbas, and H. H. J. ten Kate
}

\begin{abstract}
Cyclic energizing of a magnet coil with Cable in Conduit Conductors (CICC), as for fusion applications, results in an anomalous change of the interstrand contact resistance $\left(R_{c}\right)$ and coupling loss $(n \tau)$ due to the alternating transverse forces. Previously, three $\mathrm{Nb}_{3} \mathrm{Sn}$ ITER conductors have been tested in a cryogenic press, up to 40 cycles. Now, for the first time, the behavior of $\mathrm{NbTi}$ conductors under cyclic load is investigated and results are presented for three full-size prototype ITER Poloidal Field (PF) Coil conductors. One conductor has bare copper strands and no petal wrapping while the others have a $\mathrm{Cr}$ and solder strand surface coating. The press can transmit a maximum transverse force of $800 \mathrm{kN} / \mathrm{m}$ directly to a cable section of $400 \mathrm{~mm}$ length at $4.2 \mathrm{~K}$. Each conductor is tested up to $220 \mathrm{kN} / \mathrm{m}$ and 40,000 full loading cycles. The magnetization of the conductors and the $R_{c}$ between combinations of strands and strand bundles is measured at various number of cycles. It appears that the $R_{c}$ can vary for up to orders of magnitude during cyclic loading.
\end{abstract}

\section{INTRODUCTION}

$\mathbf{T}$ HE ITER CICC conductors [1] are subjected to heavy transverse loading due to the Lorentz forces in the coils causing a deformation of the cable inside the conduit. This deformation is interrelated with mechanical, electromagnetic and thermohydraulic effects. A cryogenic cable press was built at the University of Twente to simulate the impact of the Lorentz forces on a conductor equivalent to ITER magnet operating conditions [2]. The press can transmit a variable transverse (cyclic) force of more than the Central Solenoid conductor peak load $(650 \mathrm{kN} / \mathrm{m})$ directly to a cable section of $400 \mathrm{~mm}$ at $4.2 \mathrm{~K}$. The evolution of the magnetization (coupling loss time constant $n \tau$ ) and the $R_{c}$ between various strands and strand bundles inside the cable can be followed along its loading history, starting at virgin condition. Knowledge of the evolution of the $R_{c}$ is crucial for evaluation of the coupling current loss, the stability, the ability of current redistribution and the cable axial voltage-current trace in combination with the nonuniformity of the joints [3]. In previous experiments with the press, two Central Solenoid Model Coil conductors (CS1 and CS2) and a TFMC (Toroidal Field) conductor were tested, all containing $\mathrm{Nb}_{3} \mathrm{Sn}$ strands [4], [5]. It was observed that $n \tau$ declines and $R_{c}$ increases substantially with the number of cycles and it seemed that after roughly 40 cycles saturation in $R_{c}$ was reached.

Manuscript received August 6, 2002. The investigations are part of NET Contract 99/502 between the EU and the University of Twente.

The authors are with the University of Twente, Department of Applied Physics, 7500 AE Enschede, The Netherlands (e-mail: a.nijhuis@tn.utwente.nl).

Digital Object Identifier 10.1109/TASC.2003.813087
TABLE I

SPECIFICATIONS OF THE NBTi CONDUCTORS

\begin{tabular}{lll}
\hline & EM/CRPP bare Cu & VNIIKP Cr / solder \\
\hline No of strands & 1152 & 1152 \\
Twist pitch strand [mm] & 18 & 12 \\
Diameter strand [mm] & 0.81 & 0.81 \\
Cabling stages & $3 \times 4 \times 4 \times 4 \times 6$ & $3 \times 4 \times 4 \times 4 \times 6$ \\
Cabling pitches [mm] & $45 \times 85 \times 125 \times 160 \times 410$ & $51 \times 86 \times 121 \times 156 \times 407$ \\
Subcable Wrapping & Non & SS tape \\
Jacket material & SS type 1.4429 & Incoloy 908 \\
Bundle void fraction [\%] & 38 & 36 \\
\hline
\end{tabular}

The interstrand contact surfaces interfere by micro-sliding, which results in friction and anomalous $R_{c}$ behavior versus applied load [6]. It seemed possible that the $R_{c}$ could reduce again substantially after a significant number of micro-sliding cycles [7]. In order to examine this effect for ITER type of conductors, the press was modified recently for fully automatic cycling.

\section{CONDUCTOR SAMPleS}

Three full size prototype ITER NbTi CICC's are selected for the first series of measurements with 40000 full loading cycles. The layout is practically similar to that of the CS1 conductor [1]. One conductor is manufactured by Euro Metalli (EM, Italy) [8] and the others are manufactured by the Russia Joint Stock Company VNIIKP Moscow (Russia). The EM CICC has no high resistive petal (last minus one cabling stage) wrapping and no strand coating and was tested in SULTAN at Centre de Recherches en Physique des Plasmas CRPP (CH). Both VNIIKP CICC's contain petal wraps, one having the strand surface coated with $\mathrm{Cr}$ and the other with solder. The solder ( $\mathrm{PbSnSb}$ ) coated conductor is identified as FS-1152 S and the $\mathrm{Cr}$ coated conductor as FS $1152 \mathrm{C}$. The samples are specified in. After splitting the conduit, the void fraction is obviously determined by the press. The AC loss of the conductors in the press is monitored by a magnetization pick-up coil method [2]. The magnetization system is calibrated by means of boil-off AC loss measurements.

The $R_{c}$ is measured with the four-point-method, using a current of $50 \mathrm{~A}$ and is defined as:

$$
R_{c}=\frac{V}{I} \cdot l[\Omega \mathrm{m}]
$$

in which $V$ is the measured voltage, $I$ is the applied current through the selected strand or strand bundle combination and $l$ is the length of the jacketed section. 


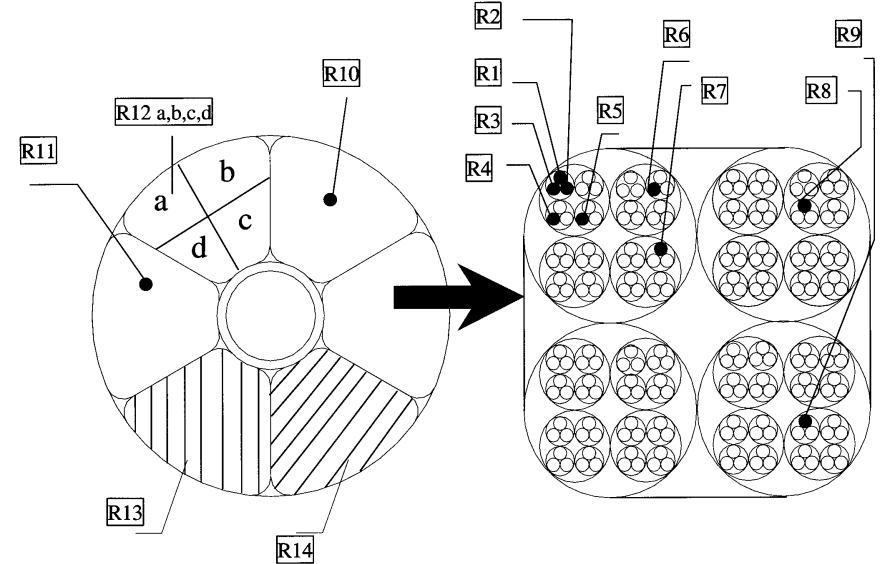

Fig. 1. Schematic representation of the strand and strand bundle connections for $R_{c}$ measurements.

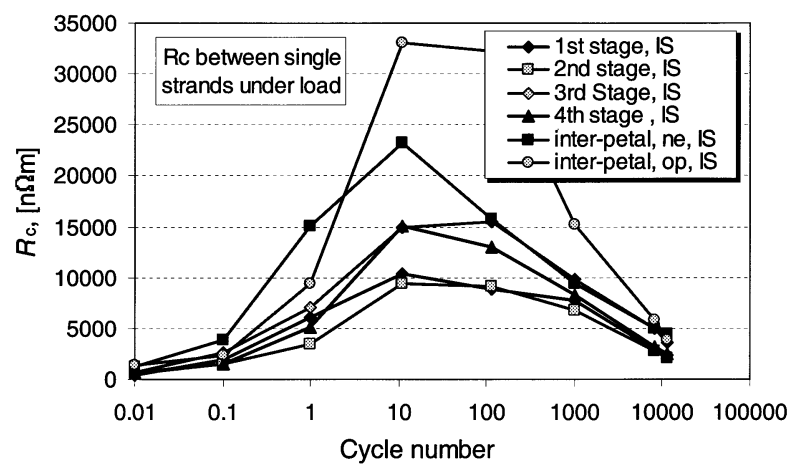

Fig. 2. $\quad R_{c}$ for single strand (IS) combinations from different cabling stages (EM/CRPP) versus number of cycles, with $F=220 \mathrm{kN} / \mathrm{m}, B_{\mathrm{dc}}=0 \mathrm{~T}$ (ne $=$ neighboring, op $=$ opposite strands or bundles).

The $R_{c}$ between a pair of sub-cables or strands is measured according to the network connections as schematically presented in Fig. 1. The connections indicated with $R 1-R 2 \ldots R 11$ refer to single strands (IS) from different locations in the cross section. While R12a-d, R13 and R14 are strand bundles for inter-bundle (IB) $R_{c}$ measurements. For these strand bundles (IB) all strands belonging to the bundle are soldered in parallel at one end.

\section{EM/CRPP CONDUCTOR Without PETAL WRAPS}

The $n \tau$ of the EM/CRPP conductor is determined only in the virgin state and amounts to $135 \mathrm{~ms}$. The transverse peak load for the NbTi conductors is chosen as $220 \mathrm{kN} / \mathrm{m}$. This is roughly a factor of two lower than the peak force for the $\mathrm{Nb}_{3} \mathrm{Sn}$ conductors, because the operating magnetic field is lower for the $\mathrm{NbTi}$ PF coils. After 11130 full loading cycles, a mechanical defect emerged in the cryogenic press and so it was brought back to room temperature in air environment. The results of all the interstrand (IS) intra-petal and inter-petal $R_{c}$ 's versus the number of loading cycles from the virgin state to 11381 cycles are presented in Fig. 2 (full load). A comparison of the $R_{c}$ values for triplet (IS), sub-petal (IB) and petal level (IB) under load is made in Fig. 3. No clear saturation was reached after 11381 cycles and so after repair of the press the experiment was continued proceeding up to 41581 cycles. The most characteristic $R_{c}$ results

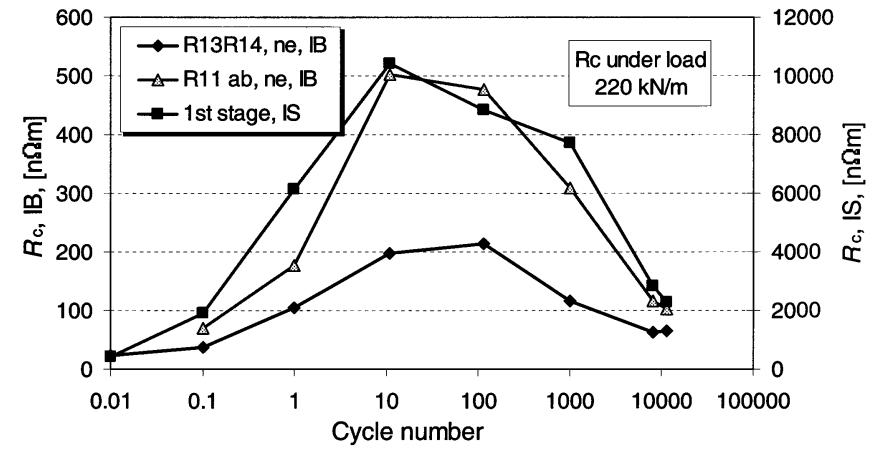

Fig. 3. The average $R_{c}$ for triplet (IS) sub-petals (IB) and petals (IB) with $F=220 \mathrm{kN} / \mathrm{m}$ versus the number of loading cycles, 1 st series.

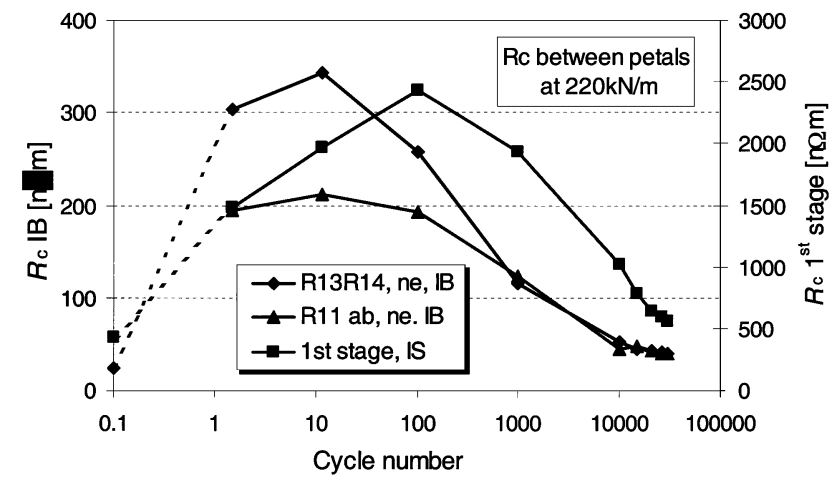

Fig. 4. The average $R_{c}$ for triplet (IS) sub-petals (IB) and petals (IB) with $F=220 \mathrm{kN} / \mathrm{m}$ versus the number of loading cycles, 2nd series.

TABLE II

$R_{c}$ Results in Fist Stage Triplet, Sub-Petal and Petal Strand BundLES UNDER Full AND ZERO LOADS, FrOM ZERO TO 41581 CyCles

\begin{tabular}{|c|c|c|c|c|c|}
\hline \multicolumn{6}{|c|}{$R_{\mathrm{c}}[\mathrm{n} \Omega \mathrm{m}]$ for neighbouring strands and bundles } \\
\hline \multirow[b]{2}{*}{ stage } & \multirow[t]{2}{*}{ virgin } & \multicolumn{2}{|c|}{$220 \mathrm{kN} / \mathrm{m}$} & \multicolumn{2}{|c|}{$0 \mathrm{kN} / \mathrm{m}$} \\
\hline & & $\max$. & final & $\max$. & final \\
\hline ( $1^{\text {st }}$ stage triplet) IS & 420 & 10,400 & 560 & 123,000 & 7,000 \\
\hline sub-petal ( $4^{\text {th }}$ stage $)$ IB & $<70$ & 500 & 39 & 1,190 & 220 \\
\hline petal ( $5^{\text {th }}$ stage) IB & 24 & 210 & 39 & 900 & 730 \\
\hline
\end{tabular}

the 2nd series are gathered in Fig. 4 and all results are summarized for both series of cycling in Table II. A relaxation in the $R_{c}$ after unloading for a one-month period is clearly observed. The maximum in $R_{c}$, under full load, for both series appears already between 10 and 100 cycles, just as it was found for the Cr-plated $\mathrm{Nb}_{3} \mathrm{Sn}$ CS and TF CICC's. The increase is about one order of magnitude compared to the virgin level, which apparently is the minimum value. The data in 'virgin state' are taken before separating the jacket because some effect on conductor handling cannot be avoided when inserting the sample into the press. Beyond the peak, between 100 and 10000 loading cycles, the $R_{c}$ gradually decreases and saturates after $10000 \mathrm{cy}-$ cles toward roughly again the virgin level. In the second series, again the same characteristic behavior is observed. The relatively strong stresses in the cable, during the cable manufacture, could possibly be responsible for creation of low resistive contacts in the virgin state. The drastic increase in $R_{c}$ during the first 100 cycles, may then be related to disruption of the low resistive contacts due to strand micro-sliding. During relaxation 


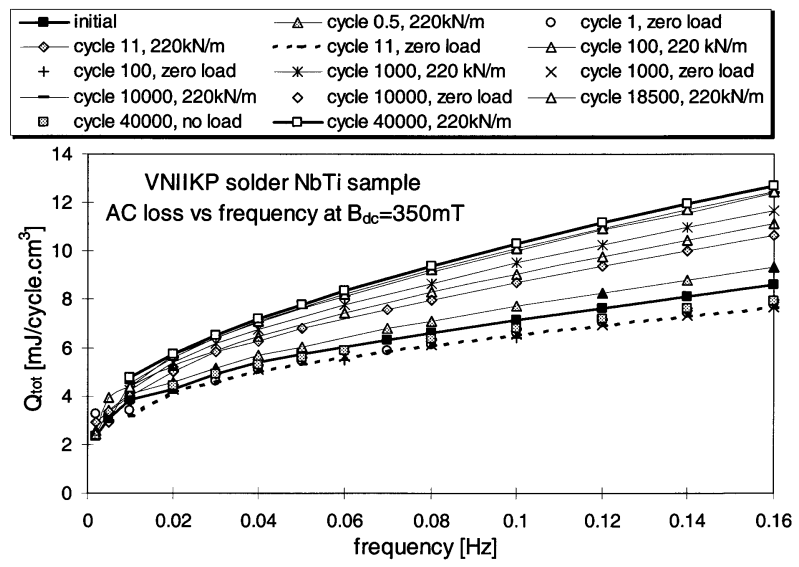

Fig. 5. Evolution of the AC loss in the FS 1152-S specimen in initial state, under load $(220 \mathrm{kN} / \mathrm{m})$ and without load up to 40000 cycles.

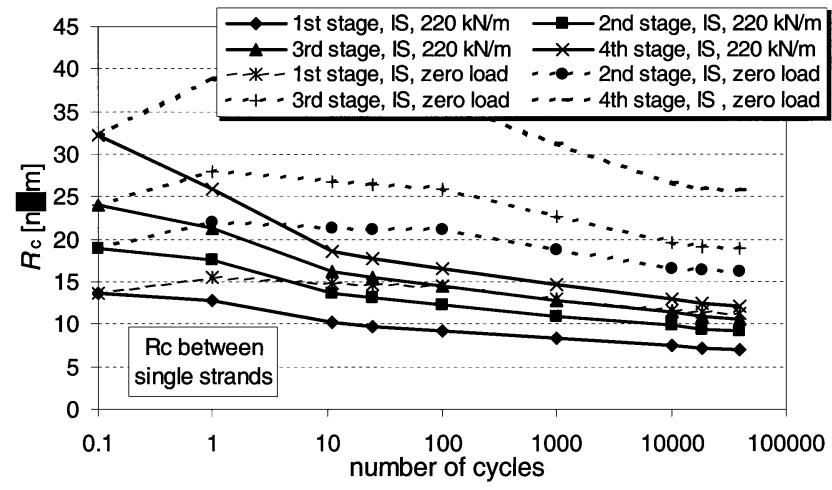

Fig. 6. $R_{c}$ for single strand combinations (IS) from different cabling stages (FS $1152-\mathrm{S}$ ) versus cycling, $F=0$ and $220 \mathrm{kN} / \mathrm{m}, B_{\mathrm{dc}}=0.35 \mathrm{~T}$.

of the cable in between the two series of measurements, the $R_{c}$ might be increased due to oxidation of the polished spots or due to further dislocation of the strand positions.

\section{REsults SOLDER COATED CICC (FS1152-S)}

\section{A. AC Loss Measurements on FS 1152-S}

The AC loss results obtained by magnetization on the solder coated VNIIKP conductor FS 1152-S are shown in Fig. 5. Every time when releasing the load along cycling, the loss returns to a level, which is slightly below what was found in the 'initial state' and does not further depend too much on the loading history (dotted line). The 'initial state' of the sample, after insertion in the press, is not equal to 'virgin' as some handling of the conductor is required after releasing the clamps from the splitted conduit halves. Nevertheless, for this AC loss case also a virgin specimen was measured. Under full load, the loss increases with the number of cycles but saturates when approaching 10000 cycles. The loss versus frequency behaves strongly nonlinear below $0.05 \mathrm{~Hz}$ but seems rather linear above $0.05 \mathrm{~Hz}$. For frequencies higher than $0.05 \mathrm{~Hz}$ the $n \tau$ amounts to $170 \mathrm{~ms}$ in the virgin state.

\section{B. Contact Resistance Measurements on FS 1152-S}

The $R_{c}$ strongly depends on the background field as the solder is superconducting below $0.2 \mathrm{~T}$. Hence a DC field of

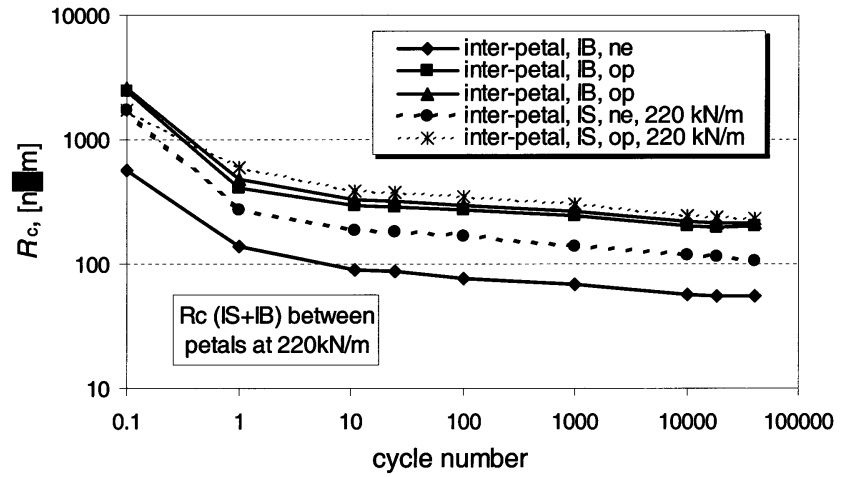

Fig. 7. Interstrand (IS) and interbundle (IB) $R_{c}$ for petal combinations (FS-1152 S) versus number of cycles, with $F=220 \mathrm{kN} / \mathrm{m}, B_{\mathrm{dc}}=0.35 \mathrm{~T}$.

TABLE III

$R_{c}$ Results in 1st Stage Triplet, Sub-Petal and Petal Strand Bundles UNDER FULl AND ZERO LOADS, From ZERO TO 40000 CYCLES

\begin{tabular}{cccccc}
\hline \multicolumn{4}{c}{$R_{\mathrm{c}}[\mathrm{n} \Omega \mathrm{m}]$ for neighbouring strands and bundles } \\
\hline \multicolumn{4}{c}{$220 \mathrm{kN} / \mathrm{m}$} \\
\hline stage & max. & end & initial & max. & end \\
\hline$\left(^{\text {st }}\right.$ stage triplet) IS & 14 & 7 & 14 & 15 & 11 \\
Sub-petal ( $4^{\text {th }}$ stage) IB & 8 & 2.2 & 8 & 10 & 7 \\
petal $\left(5^{\text {th }}\right.$ stage) IB & 570 & 55 & 570 & 1080 & 550 \\
\hline
\end{tabular}

$0.35 \mathrm{~T}$ is required to keep the solder permanently in the normal state. The results of all the interstrand (IS) intra-petal $R_{c}$ 's for full load and zero load, versus the number of loading cycles from the initial state to 40000 cycles, are presented in Fig. 6 . The $R_{c}$ within a petal decreases gradually by a factor of two within 40000 cycles. The sub-petal inter-bundle $R_{c}$ (IB) under load reduces by a factor of three to four within 40000 cycles. Without load there is hardly any change in the $R_{c}$ with cycling. The evolution of the inter-petal $R_{c}$ for single strand combinations (IS) and full bundle combinations (IB) under load is compared in Fig. 7. The major part of the reduction in $R_{c}$ occurs during the first loading cycles and amounts to one order of magnitude, while there is hardly any difference between IS and IB. The most important results from the evolution of the $R_{c}$ versus cycling are summarized in Table III. The maximum $R_{c}$ under full load is already in the initial state and then gradually decreases with cycling. Without load the $R_{c}$ passes a maximum and decreases again after 100 cycles.

The final saturation level of $R_{c}$ (under load) is reached after 10000 cycles and is plainly below that in the initial state, in contradiction to the EM/CRPP conductor with bare copper strands. The effect of transverse cable loading on the $R_{c}$ for intra-petal combinations with solder coated strands is moderate. Still, for the inter-petal $R_{c}$ changes up to one order of magnitude with cycling are observed under load. It is obvious that the coupling loss is mainly generated inside the petals and this is well in agreement with the measured results. The level of coupling loss with and without load corresponds well to the intra-petal $R_{c}$. Analysis of the $n \tau$ and $R_{c}$ in the virgin, initial and final state, suggests that the virgin $R_{c}$ should be roughly similar to the final $R_{c}$. And so the conductor handling likely already caused an increase in $R_{c}$. 


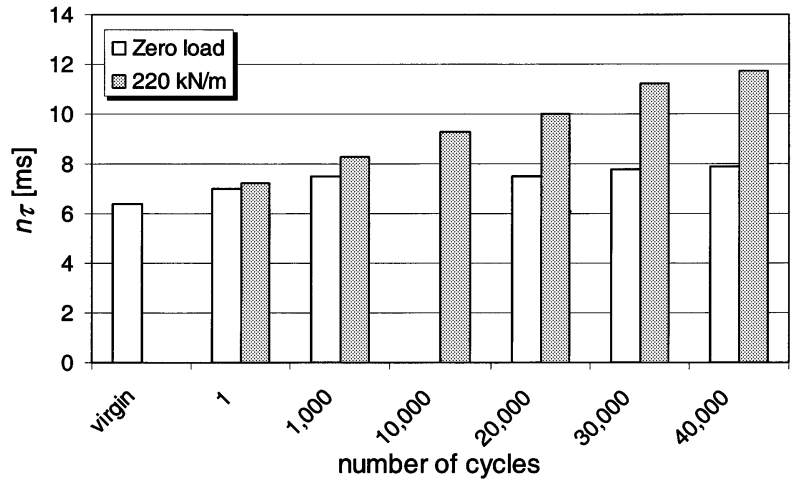

Fig. 8. The AC loss of the FS1152-S specimen in the virgin state, under load $(220 \mathrm{kN} / \mathrm{m})$ and without load up to 40000 cycles.

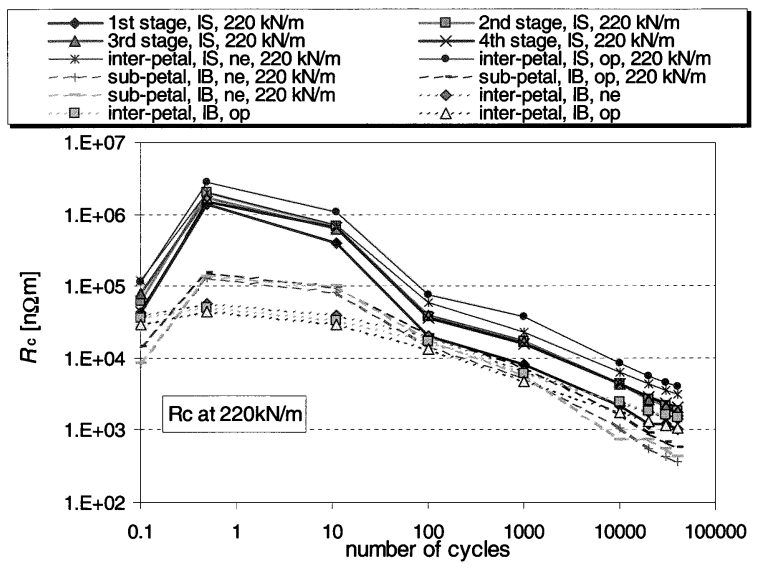

Fig. 9. $\quad R_{c}$ for single strand combinations (IS) from different cabling stages (FS $1152-\mathrm{C}$ ) versus cycle number, $F=220 \mathrm{kN} / \mathrm{m}, B_{\mathrm{dc}}=0.35 \mathrm{~T}$.

\section{Results Chromium COATED CICC (FS1152-C)}

\section{A. AC Loss Measurements on FS 1152-C}

The coupling loss time constants obtained on the chromium coated VNIIKP conductor are shown in Fig. 8. The coupling loss is mainly from intra-strand origin but with cycling an additional interstrand component is coming up.

\section{B. Contact Resistance Measurements on FS 1152-C}

The results of all the IS and IB, petal and sub-petal $R_{c}$ 's for full load versus the number of cycles are presented in Figs. 9, 10 and Table IV (Note that the $R_{c}$ in Table IV is in $\mu \Omega \mathrm{m}$ instead of $\mathrm{n} \Omega \mathrm{m}$ ). The evolution of the $R_{c}$ without load is not presented because it is similar to the case with load except that the level is about one order of magnitude higher. The $R_{c}$ reaches a maximum already before ten cycles, after which it gradually decreases for three orders of magnitude without showing clear saturation after 40000 cycles. The final $R_{c}$ level is not far from what is observed for the previously tested $\mathrm{Nb}_{3} \mathrm{Sn}$ CS and TF CICC's [5]. Besides electromagnetic forces, the strands need sufficient freedom to move and so the void fraction might play a significant role.

\section{SUMMARY}

Cyclic cable loading, simulating electromagnetic forces for NbTi ITER type of CICC's, can have a severe impact on the in-

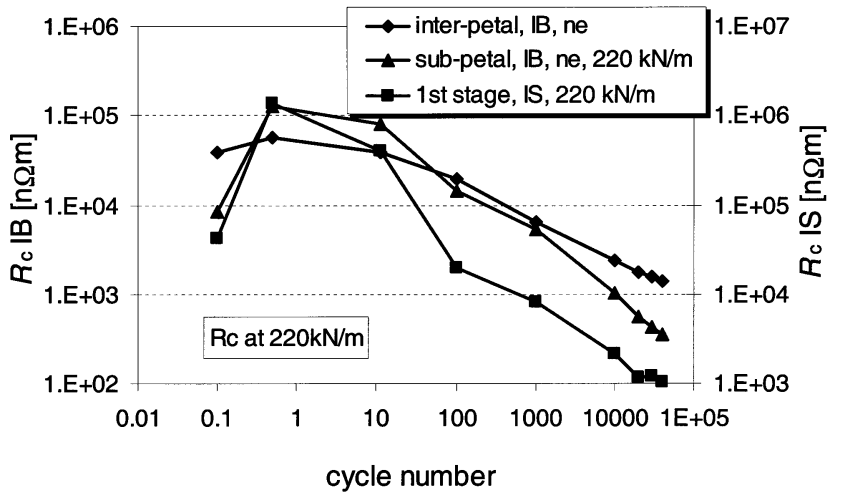

Fig. 10. The average $R_{c}$ for triplet (IS), sub-petals (IB) and petals (IB) with $F=220 \mathrm{kN} / \mathrm{m}$ versus the number of loading cycles, (FS 1152-C).

TABLE IV

$R_{c}$ Results in 1St Stage Triplet, Sub-Petal and Petal Strand Bundles UNDER FULL AND ZERO LOADS FROM ZERO TO 40000 CYCLES

\begin{tabular}{|c|c|c|c|c|c|}
\hline \multicolumn{6}{|c|}{$R_{\mathrm{c}}[\mu \Omega \mathrm{m}]$ for neighbouring strands and bundles } \\
\hline \multirow[b]{2}{*}{ stage } & \multicolumn{2}{|c|}{$220 \mathrm{kN} / \mathrm{m}$} & \multirow[b]{2}{*}{ vigin } & \multicolumn{2}{|c|}{$0 \mathrm{kN} / \mathrm{m}$} \\
\hline & $\max$. & end & & $\max$. & end \\
\hline ( $1^{\text {st }}$ stage triplet) IS & 1350 & 1.0 & 43 & 4160 & 5.0 \\
\hline Sub-petal ( $4^{\text {th }}$ stage) IB & 129 & 0.36 & 8.5 & 470 & 1.6 \\
\hline petal $\left(5^{\text {th }}\right.$ stage $)$ IB & 57 & 1.42 & 39 & 200 & 7.4 \\
\hline
\end{tabular}

terstrand contact resistance $R_{c}$. There is a tendency for increase of $R_{c}$ along the first decade of loading cycles. For bare copper strands the maximum in $R_{c}$ is one order of magnitude above the virgin $R_{c}$. Beyond the peak, the $R_{c}$ saturates toward again that of the virgin state. The $R_{c}$ within a petal of a CICC with solder coated strands changes up to a factor of two within $40000 \mathrm{cy}-$ cles. The Cr-plated specimen reaches its maximum $R_{c}$ already during the first cycle and then decreases with three orders of magnitude.

\section{REFERENCES}

[1] N. Mitchell et al., "Conductor Development for the ITER Magnets," in MT-15 Proc., Beijing, China, 1998, pp. 347-352.

[2] A. Nijhuis, N. H. W. Noordman, H. H. J. ten Kate, N. Mitchell, and P. Bruzzone, "A press for mechanical and electrical testing of full-size ITER conductors under transverse loading," in MT-15 Proceedings, Beijing, China, 1998, pp. 441-444.

[3] A. Nijhuis, H. G. Knoopers, Y. Ilyin, B. ter Haken, A. Godeke, and H. H. J. ten Kate, "Effect of self-field and current nonuniformity on the voltage-temperature characteristic of the ITER central solenoid insert coil by numerical calculations," Cryogenics, vol. 42, pp. 469-483, 2002.

[4] A. Nijhuis, N. H. W. Noordman, H. H. J. ten Kate, N. Mitchell, and P. Bruzzone, "Electromagnetic and mechanical characterization of ITER CS-MC conductors affected by transverse cyclic loading, part 1: Interstrand coupling losses," IEEE Trans. on Supercond., vol. 9, no. 2, pp. 1069-1072, June 1999.

[5] — "Electromagnetic and mechanical characterization of ITER CS-MC conductors affected by transverse cyclic loading, part 2: Interstrand contact resistances," IEEE Trans. on Supercond., vol. 9, no. 2, pp. 754-757, June 1999.

[6] A. Nijhuis and H. H. J. ten Kate, "Surface oxidation and interstrand contact resistance of $\mathrm{Cr}$-coated $\mathrm{Nb}_{3} \mathrm{Sn}$ and bare NbTi strands in CICC's," Adv. in Cryog. Eng., vol. 46B, pp. 1083-1090, 2000.

[7] A. Iwabuchi et al., "The effects of coated film on (NbTi) ${ }_{3} \mathrm{Sn}$ wire on friction and electric contact resistance," in Proc. of ICEC/ICMC-16 Conf., Japan, May 1996, pp. 1727-1730.

[8] B. Blau, "Stability and Quench of Dual Cooling Channel Cable-in-Conduit Superconductors," Ph.D., CRPP, Villigen, CH, 1999. 